\title{
A randomised trial of probiotics to reduce severity of physiological and microbial disorders induced by partial gastrectomy for patients with gastric cancer
}

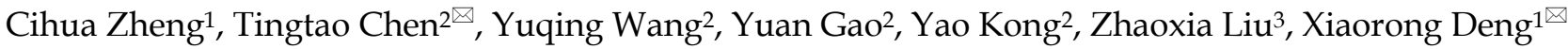 \\ 1. Department of Gastrointestinal Surgery, The Second Affiliated Hospital of Nanchang University, Nanchang, Jiangxi 330006, PR China. \\ 2. Institute of Translational Medicine, Nanchang University, Nanchang, Jiangxi 330031, PR China \\ 3. Department of Obstetrics and Gynecology, The Second Affiliated Hospital of Nanchang University, Nanchang, Jiangxi 330006, PR China.
}

$\square$ Corresponding authors: Dr. Xiaorong Deng, Department of Gastrointestinal Surgery, The Second Affiliated Hospital of Nanchang University, 1 Minde Road, Nanchang, Jiangxi 330006, P.R. China. E-mail: dengxr77@163.com. Dr. Tingtao Chen, Institute of Translational Medicine, Nanchang University, 1299 Xuefu Road, Nanchang, Jiangxi 330031, P.R. China; e-mail: chentingtao1984@163.com

(c) Ivyspring International Publisher. This is an open access article distributed under the terms of the Creative Commons Attribution (CC BY-NC) license (https:// creativecommons.org/licenses/by-nc/4.0/). See http://ivyspring.com/terms for full terms and conditions.

Received: 2018.08.07; Accepted: 2018.10.31; Published: 2019.01.01

\begin{abstract}
Gastrectomy has been widely used for the treatment of gastric cancer, and the severity of physiological and microbial disorders has greatly harmed the health of patients. In the present study, a probiotic combination containing Bifidobacterium infantis, Lactobacillus acidophilus, Enterococcus faecalis and Bacillus cereus was used to reduce the physiological disorders induced by gastrectomy via monitoring the blood index and microbial diversity using high-throughput sequencing. Our results indicated that the probiotic combination had significantly reduced the inflammation indexes (leukocyte) $(p<0.05)$, while it markedly enhanced the immunity indexes (lymphocyte) and nutrition indexes (albumin and total protein) $(p<0.05)$. In addition, gastric cancer had a strong influence on the microbial diversity of the stomach via enhancing the number of pathogens of Streptococcus, Peptostreptococcus and Prevotella, and reducing the percentage of the probiotic Bifidobacterium. Although partial gastrectomy markedly changed intestinal microbial diversity, the taking of the probiotic combination greatly reduced the ratio of Firmicutes/Bacteroidetes compared with patients taking no probiotics at the phylum level. At the genus level, the probiotic combination significantly enhanced the numbers of the probiotic bacteria Bacteroides, Faecalibacterium and Akkermansia and lowered the richness of Streptococcus. Therefore, we concluded that the taking of the probiotic combination significantly enhances the immune response of patients and reduces the severity of inflammation through modification of gut microbiota.
\end{abstract}

Key words: Gastric cancer, gastrectomy, probiotics, high-throughput sequencing, Akkermansia

\section{Introduction}

Gastric cancer is developed from the lining of the stomach and is regarded as the leading cause and death of cancers [1,2]. Gastric cancer can occur as a result of many factors, such as infections, smoking, diet and genetics [3], and the symptoms of bloating, weakness of the stomach, diarrhoea/constipation, abdominal pain, weight loss and bleeding caused by this disease have greatly lowered the quality of life of patients [4].

Until now, surgery, radiation therapy, chemotherapy, gene therapy and immunotherapy have been used for the treatment of gastric cancer, among which gastrectomy is the most common surgical procedure for gastric cancer patients [5-7]. However, the total/partial removal of the stomach has caused the loss of a storage place for food, and only a small amount of food can be allowed into the small intestine at a time, leading to postoperative symptoms such as dysphagia, heartburn and nutritional disorders [8-10]. Therefore, it is a key point to enhance the quality of life of patients after gastrectomy.

As we know, the microbiota play a crucial role in disease development and prevention, and its role in 
human life has gained much importance in recent years [11]. There are about 100 trillion commensal microbes in the human body, which represents a 100 -fold multiplication of the genes in the human genome, and the interactions between microbiota and host immunity system (affecting susceptibility against immune-mediated and infectious diseases) bring new and interesting therapeutic approaches for various diseases [12-16]. Until now, many studies have revealed the roles of microbiota in cancers, neuropathic diseases, obesity, diabetes and gastrointestinal diseases [17-21], and our previous work also indicated that probiotic combinations had a sound effect on severity of OM (oral mucositis) which was a common unpreventable complication induced by radiochemotherapy in NPC (nasopharyngeal carcinoma) patients with CCRT (concurrent radiochemotherapy), via enhancing the immune response of patients and reducing the severity of $\mathrm{OM}$ through modification of gut microbiota (data not shown). However, no study has been carried out to explore the intervention of probiotics in gastric cancer patients after gastrectomy.

In the present study, the probiotic combination of Bifidobacterium infantis, Lactobacillus acidophilus, Enterococcus faecalis and Bacillus cereus was supplied to gastric cancer patients with partial gastrectomy, and their effect on reducing the severity of physiological disorders and microbial diversity in the stomach and faeces were investigated.

\section{Materials and methods}

\section{Study design and patient enrolment}

The randomised trial was conducted at the Second Affiliated Hospital of Nanchang University in China. The trial enrolled male and female patients aged between 37 and 79 years with gastric cancer between January 2017 and April 2018. All patients received a series of medical evaluations, including medical history, physical examination, blood test, abdominal ultrasound, and chest computed tomography (CT) scan before enrolment (Table 1). All patients received no radiotherapy or/and chemotherapy before the treatment, and only patients treated with partial gastrectomy were enrolled for the experimental consistency. Three to five days after the partial gastrectomy, patients resumed diets (e.g., noodles or porridge) together with the probiotic for another 7 days.

In addition, the gastric juices obtained from 5 healthy people used in microbial diversity analysis were named as the HS group. Patients with the following features were excluded: immune diseases, under high-risk for antimicrobial agents (e.g., diabetes or senile dry stomatitis), inability to take medicine orally or absorb medicine in the digestive tract, previous cancer or coexisting tumours or allergic to probiotics. Patient samples and data were obtained with written informed consent in accordance with the ethics committee requirements at the participating institute and the Declaration of Helsinki. Permission to carry out the study was obtained from the Institutional Review Board (IRB) of the Second Affiliated Hospital of Nanchang University.

\section{Trial protocol}

Patients were randomly distributed to two blocks in a 1:1 ratio to receive probiotics or placebo. The drug was distributed and packaged in accordance with random numbers, and the blinding codes were not disclosed during the whole trial period.

Probiotic (Bifidobacterium Tetravaccine Tablets, SFDA approval number: S20060010, containing $>10^{6}$ $\mathrm{CFU} /$ table $B$. infantis, > $10^{6} \mathrm{CFU} /$ table L. acidophilus, > $10^{6} \mathrm{CFU} /$ table E. faecalis, $>10^{5} \mathrm{CFU} /$ table B. cereus and $>10^{6} \mathrm{CFU} /$ table total bacteria) or placebo was supplied 3-5 days after partial gastrectomy for up to 6-7 days (three capsules, three times a day). If these patients had any side effects generated by the probiotic combination, the side effect would have been followed up until it disappeared. Venous blood samples were obtained by venepuncture in the antecubital fossa without excessive venous stasis. The blood samples were sampled in an EDTA bulb and processed within 3 hours of collection in an automated haematology analyser. The parameters of routine blood, biochemical analysis and lymphocyte immunity were measured before and after treatment. General physical examinations, laboratory tests, unplanned delays, vital signs and so on were completed at baseline and at the end of the study.

\section{Total bacterial genomic DNA extraction and high-throughput sequencing}

Gastric juice or faeces was sampled from health people or patients and were stored at $-70{ }^{\circ} \mathrm{C}$. The combination of genomic DNA kits (Tiangen Biotech Co., Ltd., Beijing, China) and the bead beating method were used [22-24], and the concentration and quality of purified DNA was determined via a spectrophotometer at $230 \mathrm{~nm}$ (A 230) and $260 \mathrm{~nm}$ (A 260) (NanoDrop; Thermo Fisher Scientific, Inc., Waltham, MA, USA). Then, the V4 region of the $16 \mathrm{~S}$ rDNA genes in each sample were amplified using primers of $515 \mathrm{~F} / 806 \mathrm{R}$ primers $(515 \mathrm{~F}$, 5'-GTGCCAGCMGCCGCGGTAA-3'; 806R, 5'-GGAC TACVSGGGTATCTAAT $-3^{\prime}$ ), and these PCR products were sequenced with an Illumina HiSeq 2000 platform (GenBank accession number SRP155279) [25]. 


\section{Bioinformatics and multivariate statistics}

Paired-end reads from the original DNA fragments were processed by using Cutadapt (V1.9.1, http://cutadapt.readthedocs.io/en/stable/) and the UCHIME Algorithm (http://www.drive5.com/ usearch/manual/uchime_algo.html) based on the unique barcodes [26]. Sequence analysis was subsequently performed using the UPARSE software package (version 7.0.100), and sequences with $\geq 97 \%$ similarity were assigned to the same operational taxonomic units (OTUs). Then, Qiime software (version 1.9.1) was used to analyse the a diversity (within samples, indexes of observed OTUs, Chao1, Shannon, Simpson, ACE, and goods coverage) and $\beta$ diversity (among samples, PCA, PCoA and NMDS) [27, 28]. The cluster analysis was preceded by weighted UniFrac distance using the QIIME software package (version 1.8.0) [29], partial least squares discriminate analysis (PLS-DA) was preceded by using SIMCA-P software version 11.5 (Umetrics; Sartorius Stedim Biotech, Malmö, Sweden).

All data were reported as means $\pm \mathrm{SD}$, and results were analysed using SPSS 23.0 software (SPSS Inc., Chicago, IL, USA) by means of unpaired F-tests. All tests were two-tailed, with the level of significance set at $\mathrm{p}<0.05$.

\section{Results}

\section{Patients' baseline characteristics}

Between January 2017 and April 2018, 100 patients were randomly (1:1) assigned to either the probiotic combination group (50 patients) or placebo group (50 patients).

All patients were thoroughly informed about their diseases and the treatments they would receive. Patients were randomised into two groups, and their sex, age, baseline characteristics and tumour classification are summarised in Table 1 . The treatment groups were well balanced, and there was no marked difference between AC group and ACP group.

\section{Probiotic combination reduced physiological disorders induced by partial gastrectomy}

To assess whether the probiotic combination had an effect on physiological disorders, we monitored the physiological indexes in blood of patients. We found that the probiotic combination significantly reduced the leukocyte inflammation indexes $(102.7 \%$ vs. $162.8 \%, p<0.0001)$, and significantly enhanced the lymphocyte immunity indexes $(80.31 \%$ vs. $59.79 \%$, p < $0.001)$, the nutrition indexes of albumin $(88.75 \%$ vs. $84.38 \%, \mathrm{p}<0.05)$ and total protein $(88.63 \%$ vs. $82.49 \%$, $\mathrm{p}<0.01$ ) (Fig. 1) compared with the AC group.
Table 1. Baseline patient demographics and characteristics. AC, cancer patients after gastrectomy and treatment without probiotic combination; ACP, cancer patients after gastrectomy and treatment with probiotic combination.

\begin{tabular}{llll}
\hline Variable & AC Group (N=50) & $\begin{array}{l}\text { ACP Group } \\
\text { (N=50) }\end{array}$ & P value \\
\hline $\begin{array}{l}\text { Percentage of total enrollment, } \\
\text { No. (\%) }\end{array}$ & $50(50.00)$ & $50(50.00)$ & $/$ \\
Male: female, n:n (\%:\%) & $41: 9(82.00: 18: 00)$ & $43: 7(86.00: 14.00)$ & 0.585 \\
Age, y & $64.00(56.75-69.25)$ & 60.00 & 0.122 \\
& & $(51.75-67.25)$ & \\
Tumor category, No. (\%) & & & \\
T0 & $0(0.00)$ & $1(2.00)$ & 0.510 \\
T1 & $6(12.00)$ & $3(6.00)$ & \\
T2 & $5(10.00)$ & $3(6.00)$ & \\
T3 & $9(18.00)$ & $7(14.00)$ & \\
T4 & $30(60.00)$ & $36(72.00)$ & \\
Node category, No. (\%) & & & \\
N0 & $16(32.00)$ & $16(32.00)$ & \\
N1 & $23(46.00)$ & $22(44.00)$ & \\
N2 & $6(12.00)$ & $9(18.00)$ & \\
N3 & $5(10.00)$ & $3(6.00)$ & \\
M category, No. (\%) & & & \\
M0 & $50(100.00)$ & $50(100.00)$ & \\
TNM category, No. (\%) & & & \\
TNM1 & $9(18.00)$ & $6(12.00)$ & \\
TNM2 & $13(26.00)$ & $9(18.00)$ & \\
TNM3 & $28(54.00)$ & $35(70.00)$ & \\
\hline & & & \\
\hline
\end{tabular}

Moreover, our data also indicated that the probiotic combination had obviously recovered the rate of leukocyte $(92 \%$ vs. $82 \%)$, central granulocyte (92\% vs. $40 \%)$, central granulocyte ratio $(60 \%$ vs. $34 \%)$, lymphocyte (14\% vs. $2 \%)$ and lymphocyte ratio $(32 \%$ vs. $8 \%)$, erythrocyte $(14 \%$ vs. $2 \%)$, creatinine $(72 \%$ vs. $54 \%)$ and chlorinum $(80 \%$ vs. $64 \%)$ to the normal levels compared with the control group (Table 2).

\section{Comparison of the microbial community of stomach between HS and BCS groups}

To compare the microbial diversity between HS and BCS groups, the V4 hypervariable region of bacteria was amplified using the $16 \mathrm{~S}$ rRNA amplicon sequencing method. A total of 1,014,257 filtered clean reads $(78,019.77$ reads/sample) and 6,317 OTUs were obtained from all the samples with an average of 485.92 OTUs per group (data not shown).

In Fig. 2A and Fig. 2B, the observed species and Shannon index indicated that the occurrence of gastric cancer had little effect on the a diversity of the microbial community of the stomach between the HS and BCS groups. Then, the top 10 microorganism populations at the phylum level showed that Firmicutes, Bacteroidetes, Proteobacteria and Actinobacteria constituted the most common dominant phyla in these two groups, and gastric cancer had a marked increase of Firmicutes (53.04\% vs. $34.87 \%$ ) and Actinobacteria $(9.52 \%$ vs. $4.03 \%)$, while it made a sharp reduction of Bacteroidetes $(22.60 \%$ vs. $32.22 \%)$ and Proteobacteria $(9.52 \%$ vs. $17.60 \%$ ) compared with the healthy people (Fig. 2C). At the genus level, gastric cancer greatly enhanced the 

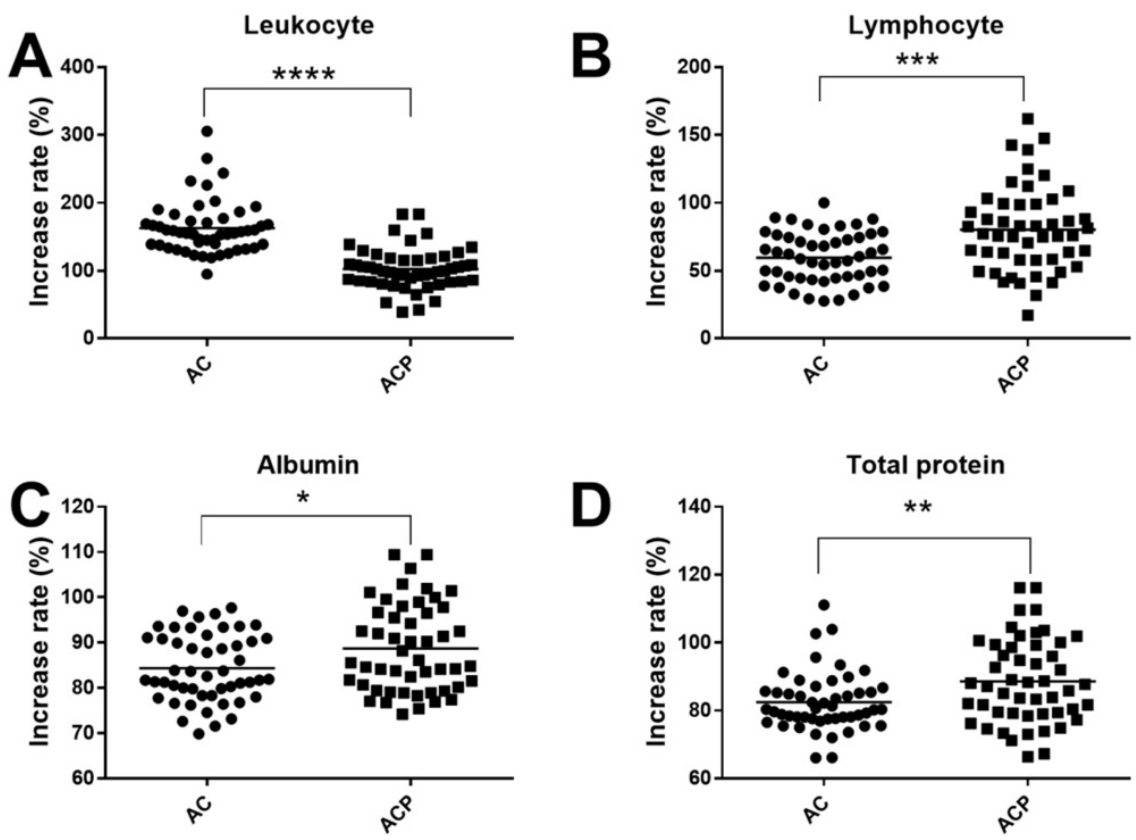

Fig. 1. Effect of probiotic combination on inflammation (A), immunity (B), and nutrition (C, D). A, Leukocyte; B, Lymphocyte; C, Albumin; D, Total protein. AC, cancer patients after gastrectomy and treatment without probiotic combination; ACP, cancer patients after gastrectomy and treatment with probiotic combination. $*$ means $p<0.05$, $* *$ means $p<0.01$, $* * *$ means $p<0.001$, and $* * * *$ means $p<0.0001$.

richness of Streptococcus $(33.60 \%$ vs. $16.49 \%)$, Peptostreptococcus (2.36\% vs. $0.16 \%)$, Prevotella $(1.35 \%$ vs. $0.6 \%$ ), while it greatly reduced the abundance of Helicobacter $(0.06 \%$ vs. $0.20 \%)$ and Bifidobacterium (0.32\% vs. $1.49 \%)$ (Fig. 2D).

Table 2. Rate of people restoring to normal range. AC, cancer patients after gastrectomy and treatment without probiotic combination; ACP, cancer patients after gastrectomy and treatment with probiotic combination.

\begin{tabular}{|c|c|c|c|c|c|}
\hline Index & $\begin{array}{l}\text { Normal } \\
\text { standards }\end{array}$ & Groups & $\begin{array}{l}\text { People } \\
\text { within } \\
\text { normal } \\
\text { range }\end{array}$ & $\begin{array}{l}\text { Total } \\
\text { people }\end{array}$ & $\begin{array}{l}\text { Rate of people } \\
\text { within normal } \\
\text { range }(\%)\end{array}$ \\
\hline \multirow[t]{2}{*}{ Leukocyte } & $(3.5-9.5) \times 10^{9}$ & $\mathrm{AC}$ & 41 & 50 & 82 \\
\hline & cells /L & $\mathrm{ACP}$ & 46 & 50 & 92 \\
\hline \multirow{2}{*}{$\begin{array}{l}\text { Central } \\
\text { granulocyte }\end{array}$} & $(2-7) \times 10^{9}$ cells & $\mathrm{AC}$ & 20 & 50 & 40 \\
\hline & $/ \mathrm{L}$ & $\mathrm{ACP}$ & 46 & 50 & 92 \\
\hline \multirow{2}{*}{$\begin{array}{l}\text { Central } \\
\text { granulocyte ratio }\end{array}$} & $(40-75) \%$ & $\mathrm{AC}$ & 17 & 50 & 34 \\
\hline & & $\mathrm{ACP}$ & 30 & 50 & 60 \\
\hline \multirow[t]{2}{*}{ Lymphocyte } & $(1.5-4) \times 10^{9}$ & $\mathrm{AC}$ & 1 & 50 & 2 \\
\hline & cells /L & $\mathrm{ACP}$ & 7 & 50 & 14 \\
\hline \multirow[t]{2}{*}{ Lymphocyte ratio } & $(20-50) \%$ & $\mathrm{AC}$ & 4 & 50 & 8 \\
\hline & & $\mathrm{ACP}$ & 16 & 50 & 32 \\
\hline \multirow[t]{2}{*}{ Hemoglobin } & $(130-175) \mathrm{g} / \mathrm{L}$ & $\mathrm{AC}$ & 4 & 50 & 8 \\
\hline & & $\mathrm{ACP}$ & 6 & 50 & 12 \\
\hline \multirow[t]{2}{*}{ Erythrocyte } & $(4.3-5.8) \times 10^{12}$ & $\mathrm{AC}$ & 1 & 50 & 2 \\
\hline & cells /L & $\mathrm{ACP}$ & 7 & 50 & 14 \\
\hline \multirow[t]{2}{*}{ Total protein } & $(65-85) \mathrm{g} / \mathrm{L}$ & $\mathrm{AC}$ & 1 & 50 & 2 \\
\hline & & $\mathrm{ACP}$ & 7 & 50 & 14 \\
\hline \multirow[t]{2}{*}{ Albumin } & $(40-55) \mathrm{g} / \mathrm{L}$ & $\mathrm{AC}$ & 1 & 50 & 2 \\
\hline & & $\mathrm{ACP}$ & 3 & 50 & 6 \\
\hline \multirow[t]{2}{*}{ Haematoblast } & $(125-350) \times 10^{9}$ & $\mathrm{AC}$ & 30 & 50 & 60 \\
\hline & cells / L & $\mathrm{ACP}$ & 33 & 50 & 66 \\
\hline \multirow{2}{*}{$\begin{array}{l}\text { Aspartate } \\
\text { aminotransfrase }\end{array}$} & $(15-35) \mathrm{U} / \mathrm{L}$ & $\mathrm{AC}$ & 33 & 50 & 66 \\
\hline & & $\mathrm{ACP}$ & 32 & 50 & 64 \\
\hline \multirow{2}{*}{$\begin{array}{l}\text { Alanine } \\
\text { aminotransfrase }\end{array}$} & $(7-40) \mathrm{U} / \mathrm{L}$ & $\mathrm{AC}$ & 49 & 50 & 98 \\
\hline & & $\mathrm{ACP}$ & 46 & 50 & 92 \\
\hline \multirow[t]{2}{*}{ Total bilirubin } & (3.4-17.1) $\mu \mathrm{mol}$ & $\mathrm{AC}$ & 40 & 50 & 80 \\
\hline & $/ \mathrm{L}$ & $\mathrm{ACP}$ & 37 & 50 & 74 \\
\hline
\end{tabular}

\begin{tabular}{llllll}
\hline Uric acid & $(155-357) \mathrm{\mu mol}$ & AC & 35 & 50 & 70 \\
& $/ \mathrm{L}$ & ACP & 40 & 50 & 80 \\
Creatinine & $(58-84) \mu \mathrm{mol}$ & AC & 27 & 50 & 54 \\
& $/ \mathrm{L}$ & ACP & 36 & 50 & 72 \\
Kalium & $(3.5-5.3) \mathrm{mmol}$ & AC & 39 & 50 & 78 \\
& $/ \mathrm{L}$ & ACP & 44 & 50 & 88 \\
Natrium & $(137-147)$ & AC & 38 & 50 & 76 \\
& mmol /L & ACP & 40 & 50 & 80 \\
Chlorinum & $(99-110) \mathrm{mmol}$ & AC & 32 & 50 & 64 \\
& $/ \mathrm{L}$ & ACP & 40 & 50 & 80 \\
& & & & & \\
\hline
\end{tabular}

In Fig. 3, the scalar-Venn results indicated that there were 1,292 and 1,028 OTUs in the HS group and BCS group, respectively, and the percentage of common OTUs was $70.36 \%(909 / 1292)$ and $88.42 \%$ (909/1028), respectively. The principal coordinates analysis (PCoA) indicated that most of the samples in the HS group clustered together, while samples in the BCS group scattered far away from the BCS groups. In addition, the Lefse analysis also indicated that Campylobacterales (at order), Prevotellaceae intermedia (at species), Helicobacteraceae (at family) and Helicobacter (at genus) were significantly higher in the HS group than in the BCS group $(\mathrm{p}<0.05)$.

\section{Effect of probiotic combination on faecal microbial diversity between BCF, ACF and ACFP groups}

When sequenced, the faecal samples of patients, $1,562,145$ filtered clean reads (78,107.25 reads/sample) and 7,853 OTUs, were obtained from all the samples with an average of 392.65 OTUs per group (data not shown), and no obvious changes of species and Shannon index were observed (Fig. 4A and Fig. 4B). 

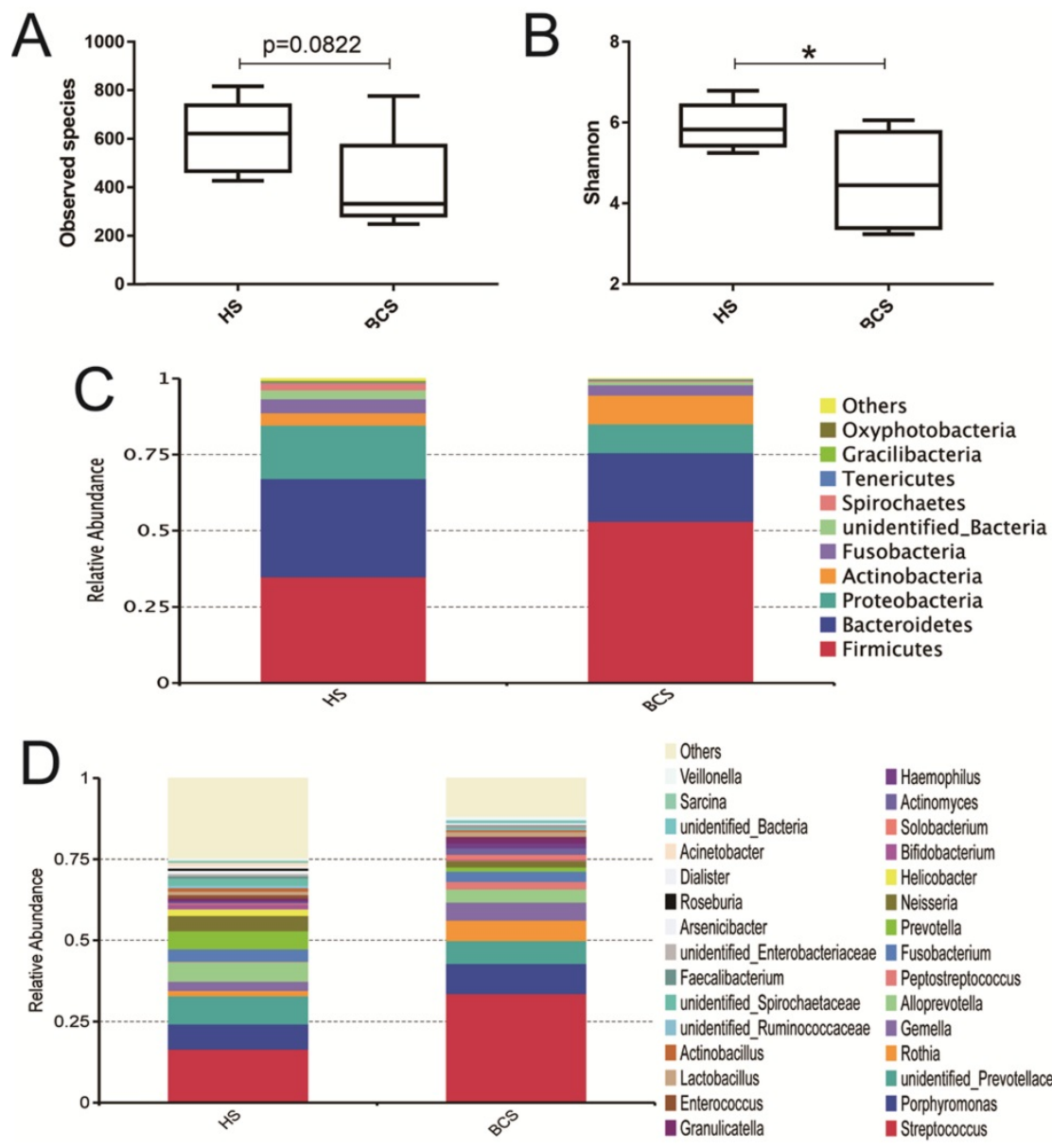

Others

Veillone
Sarcina

IInidentified_Bacteria Solobacterium

Acinetobacter Bifidobacterium

Dialister

Roseburia

Helicobacter

Arsenicibacter

Neisseria

unidentified Enterobacteriaceae Fusobacterium

Faecalibacterium Peptostreptococcus

Wunidentified_Spirochaetaceae Alloprevotella

IInidentified Ruminococcaceae Gemella

Actinobacillus

Lactobacillus

Enterococcus

Granulicatella

Innidentified_Prevotellaceae

Porphyromonas

Streptococcus

Fig. 2. Evaluation of the probiotic combination on stomach microbiota in the observed species (A), the Shannon index (B), relative abundance at the phylum level (C) and relative abundance at the genus level (D). HS, the microbiota in the stomach of healthy people; BCS, the microbiota in the stomach of patients before gastrectomy. ${ }^{*}$ means $p<0.05$.
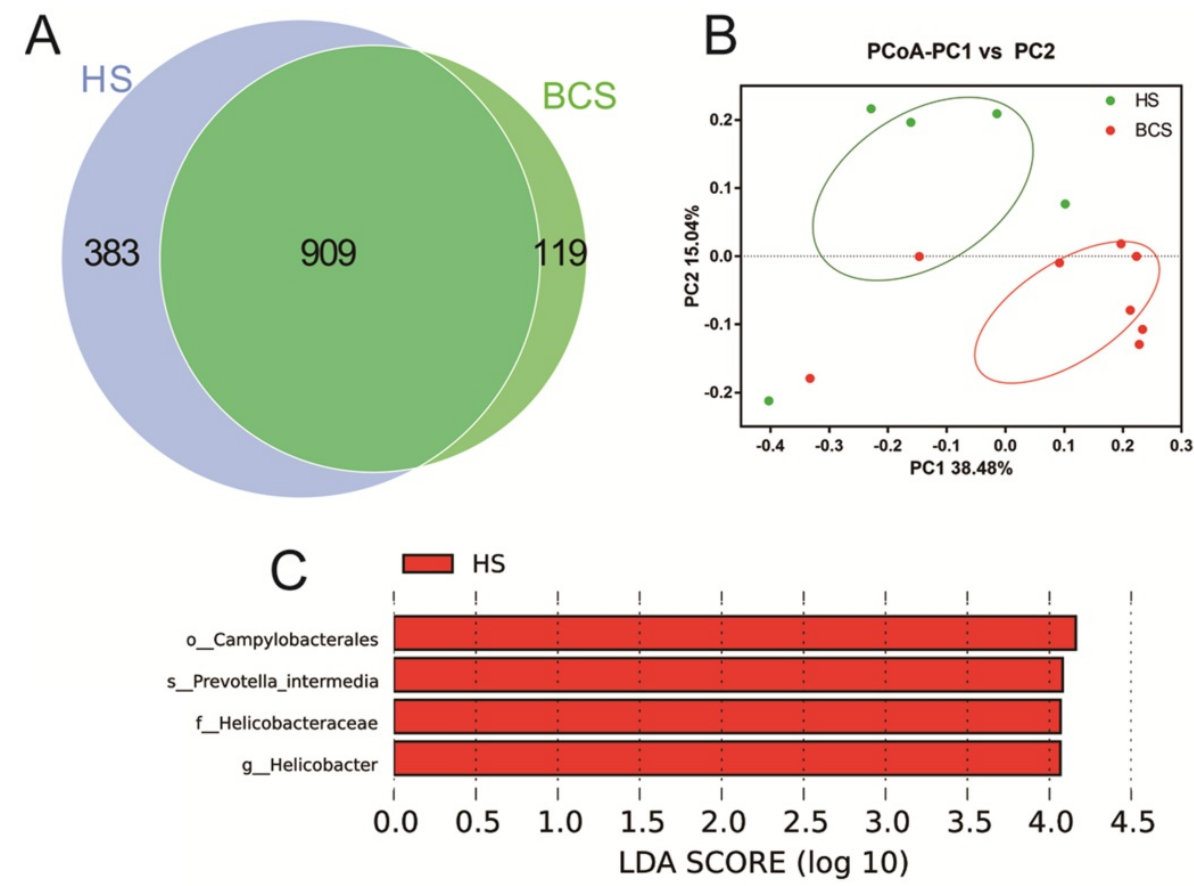

Fig. 3. Evaluation of the probiotic combination on stomach microbiota on the scalar-Venn representation (A), PCoA of $\beta$ diversity index (B) and Lefse index (C). HS, the microbiota in the stomach of healthy people; BCS, the microbiota in the stomach of patients before gastrectomy. 


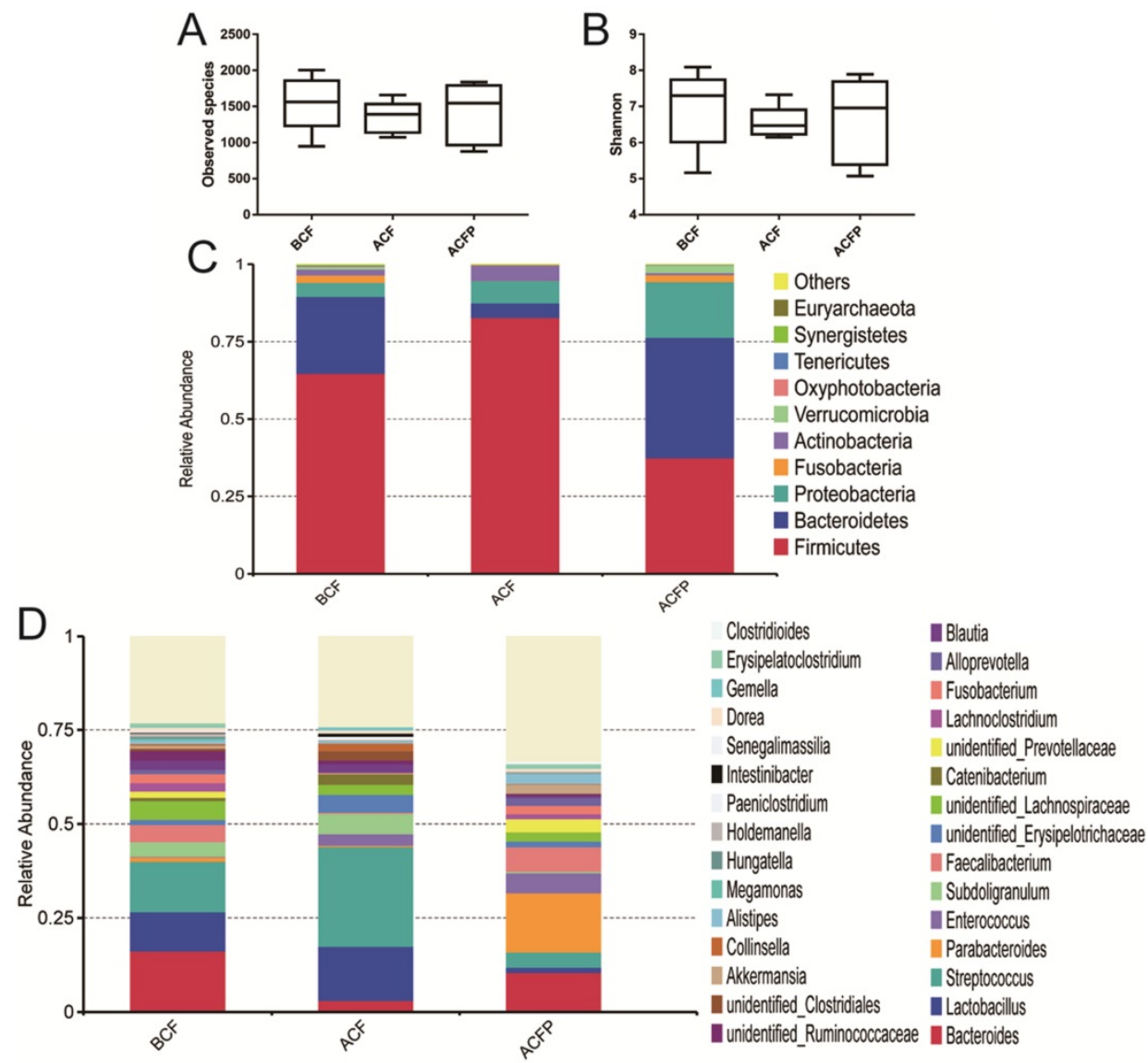

Fig. 4. Evaluation of the probiotic combination on faecal microbiota in the observed species (A), the Shannon index (B), relative abundance at the phylum level (C) and relative abundance at the genus level (D). BCF, the microbiota in faeces of patients before gastrectomy; ACF, the microbiota in faeces of patients after gastrectomy and treatment without probiotic combination; ACFP, the microbiota in faeces of patients after gastrectomy and treatment with probiotic combination.

As shown in Fig. 5, the gastrectomy seriously disturbed the microbial diversity. At the phylum level, the probiotic combination had obviously reduced the abundances of Firmicutes $(37.41 \%$ vs. $82.72 \%)$ and Actinobacteria ( $0.70 \%$ vs. $4.87 \%)$, while it markedly increased the percentage of Bacteroidetes $(38.95 \%$ vs. $4.72 \%)$, Proteobacteria ( $18.00 \%$ vs. $7.21 \%)$, Fusobacteria (2.23\% vs. $0.04 \%)$ and Verrucomicrobia $(2.42 \%$ vs. $0.01 \%)$ (Fig. 4 C). At the genus level, the probiotic combination greatly reduced the richness of Lactobacillus $(1.40 \%$ vs. $14.34 \%)$, Streptococcus $(4.00 \%$ vs. $26.58 \%)$, Subdoligranulum $(0.40 \%$ vs. $5.47 \%)$, Catenibacterium $(0.02 \%$ vs. $2.84 \%)$, and Blautia $(0.55 \%$ vs. $2.14 \%$ ), while it obviously enhanced the richness of Bacteroides $\quad(10.55 \%$ vs. $3.06 \%)$, Parabacteroides (15.75\% \% vs. 0.36$)$, Faecalibacterium ( $6.50 \%$ vs. $0.32 \%)$, Fusobacterium (2.23\% vs. 0.04\%), Alloprevotella (2.10\% vs. $0.10 \%$ ) and Akkermansia (2.42\% vs. $0.01 \%$ ).

In total, there were 5674, 3967 and 3917 OTUs in the $\mathrm{BCF}, \mathrm{ACF}$ and ACFP groups, and the common OUT number was 2067. Moreover, the samples in the BCF, ACF and ACFP groups formed clusters separated from each other, indicating that the probiotic combination had greatly changed the microbial diversity in the intestines of the host (Fig. 5).

\section{Discussion}

Gastrectomy has been widely used for the treatment of gastric cancer, while multiple factors, e.g., overgrowth of bacteria, malabsorption, and inadequate oral intake, will cause serious physiological and microbial disorders and eventually harm the health of patients. In the present study, the probiotic combination was applied to reduce the side effects caused by partial gastrectomy for patients with gastric cancer.

First, we compared the physiological indexes of blood and found that the probiotic combination had significantly reduced the inflammation indexes of leukocytes, while it markedly enhanced the immunity indexes of lymphocytes and the nutrition indexes of albumin and total protein, indicating that the probiotic combination had greatly enhanced the quality of life of patients via reducing the inflammatory state and enhancing the immunity of patients (Fig. 1, p < 0.05). In blood, the number of leukocytes is regarded as an indicator of disease, and the increase in the number of leukocytes over the upper limits possessed a strong connection with the inflammatory state of patients [30]. Moreover, the 


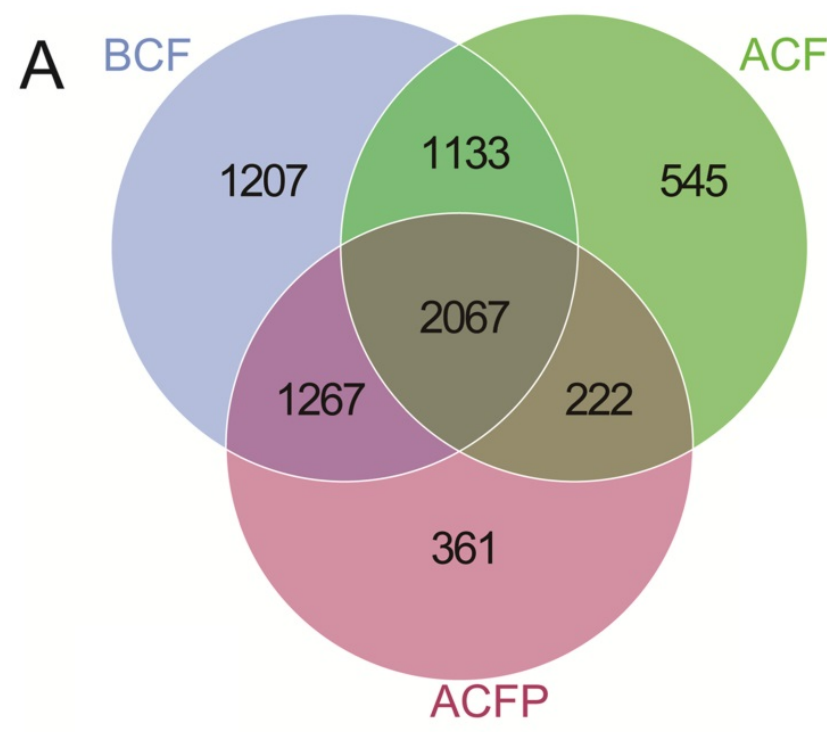

PCoA-PC1 vs PC2

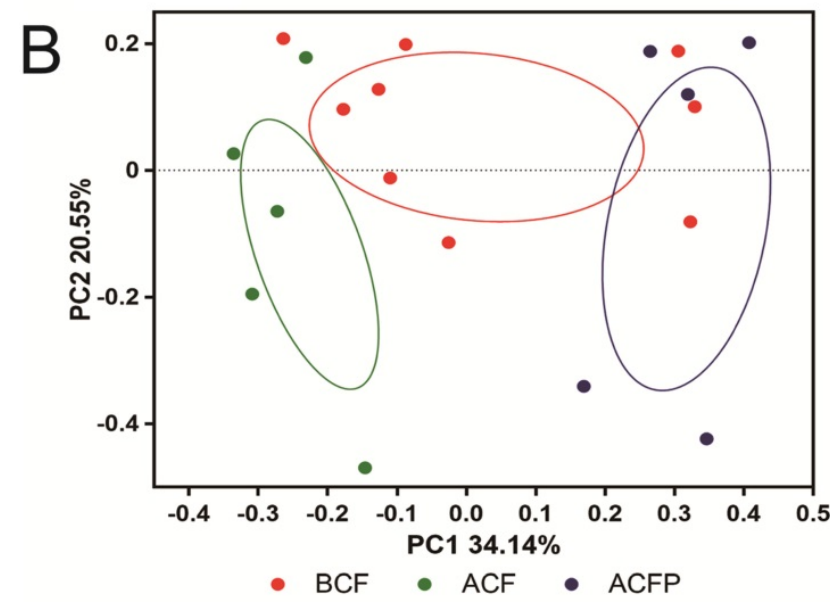

Fig. 5. Evaluation of the probiotic combination on faecal microbiota on the scalar-Venn representation (A) and PCoA of $\beta$ diversity index (B). BCF, the microbiota in faeces of patients before gastrectomy; $A C F$, the microbiota in faeces of patients after gastrectomy and treatment without probiotic combination; ACFP, the microbiota in faeces of patients after gastrectomy and treatment with probiotic combination.

enhanced number of lymphocytes, albumin and total protein indicated that the probiotic combination could help patients to enhanced nutrition and greatly enhanced the immunity of patients to help them defend against infections and promote functional recovery [31].

As we know, the human is a metaorganism consisting of host cells and a vast consortium of microbial organisms living on all of our barrier tissues. The various organisms the live in the intestines of the host provide a tremendous benefit to humans via processing of complex dietary constituents of fibre and impacts multiple organ systems (including host immunity) [32-35]. Therefore, the probiotic combination maintained the functions of host intestines, which increased the rate of leukocyte, central granulocyte and central granulocyte ratio, lymphocyte and lymphocyte ratio, erythrocyte, creatinine and chlorinum of patients in the ACP group to the normal levels (Table 2).

As the gastric cancer might have a strong influence on the microbial diversity in the stomach, the $16 \mathrm{~S}$ rRNA amplicon sequencing analysis was used to sequence the V4 hypervariable region of microbiota in the stomach. As shown in Fig. 2, gastric cancer posed little effect on species number, while it significantly changed their composition in the HS and BCS groups. Previous studies have indicated that the increased abundances of Firmicutes and the decreased abundances of Bacteroidetes had a strong connection with an unhealthy state; therefore, the increased rate of Firmicutes/Bacteroidetes in the stomachs of patients might be a potential risk to the health of patients [35-37]. Moreover, our results indicated that gastric cancer had greatly enhanced the richness of the pathogens Streptococcus, Peptostreptococcus and Prevotella, while it reduced the percentage of probiotic Bifidobacterium, confirming the harm of gastric cancer on stomach microbiota and host health. Interestingly, we also observed that gastric cancer had reduced the abundance of Helicobacter, a bacterium thought to be the causative pathogen in gastric cancer, and $2 \%$ of people with Helicobacter infections could develop stomach cancer [38].

At the end, we compared the faecal microbiota among the $\mathrm{BCF}, \mathrm{ACF}$ and $\mathrm{ACFP}$ groups and found that the use of the probiotic combination had greatly reduced the ratio of Firmicutes/Bacteroidetes compared with patients taking no probiotics, indicating that the taking of probiotics had greatly improved the intestinal microflora balance (Fig. 4). For pathogens, the probiotic combination significantly reduced the abundances of Streptococcus, while it significantly enhanced the richness of the beneficial bacteria Bacteroides and Faecalibacterium. Although the Lactobacillus in the ACFP group received a marked reduction, we found that the growth of Akkermansia, the most famous of the probiotics [39], had been significantly promoted compared with the ACP group (Fig. 4). In addition, the distinctly separate clusters of the $\mathrm{BCF}, \mathrm{ACF}$ and $\mathrm{ACFP}$ groups indicated that gastrectomy had greatly changed the microbial diversity of patients with gastric cancer and taking the probiotic combination could reduce the severity of physiological and microbial disorders via forming a new balance.

In the present study, our work showed that the probiotic combination of $B$. infantis, L. acidophilus, $E$. faecalis and $B$. cereus significantly enhanced the immunity of patients, reduced inflammation and was 
beneficial to restore the microbial diversity of patients who received gastrectomy. Therefore, the present randomised clinical trial illustrates the benefits of oral probiotics in the prevention of physiological and microbial disorders when provided to cancer patients after gastrectomy. Future studies should be devoted to explore the potential mechanisms that underlie its sound effect using animal experiments.

\section{Acknowledgements}

\section{Funding}

This work was supported by grants from the National Natural Science Foundation of China (81503364 to Tingtao Chen, and 31560264 to Tingtao Chen), the Excellent Youth Foundation of the Jiangxi Scientific Committee (20171BCB23028 to Tingtao Chen), the Science and Technology Plan of the Jiangxi Health Planning Committee (20175526 to Tingtao Chen), the Science and Technology Project of Jiangxi (20181BBG70028 to Tingtao Chen, and 20181BCB24003 to Tingtao Chen).

\section{Availability of data and materials}

All data generated or analyzed during this study are included in this published article.

\section{Authors' contributions}

$\mathrm{TC}, \mathrm{XD}$ and $\mathrm{ZL}$ designed the experiments, analyzed the data and wrote the manuscript. $\mathrm{CZ}, \mathrm{YW}$, YG, YK performed the experiments. All authors discussed the results and commented on the final manuscript.

\section{Ethics approval and consent to participate}

The protocol was approved by the Committee on the Ethics of Animal Experiments of the Second Affiliated Hospital of Nanchang University (Jiangxi, China). Patient samples were obtained with written informed consent in accordance with the Ethics Committee's requirements.

\section{Consent for publication}

Written informed consent was obtained from all participants.

\section{Competing Interests}

The authors have declared that no competing interest exists.

\section{References}

1. Cancer UK Stomach (gastric) cancer. Us National Cancer Institute. 2014

2. Stewart B, Wild C. World cancer report 2014: International Agency for Research on Cancer. 2014.

3. Lee YY, Derakhshan MH. Environmental and lifestyle risk factors of gastric cancer. Arch Iran Med. 2013; 16: 358.

4. Cancer UK. Statistics and outlook for stomach cancer. 2014
5. Pretz JL, Wo JY, Mamon HJ, Kachnic LA, Hong TS. Chemoradiation therapy: localized esophageal, gastric, and pancreatic cancer. Surg Oncol Clin N Am. 2013; 22: 511-24.

6. Meza-Junco J, Au HJ, Sawyer MB. Critical appraisal of trastuzumab in treatment of advanced stomach cancer. Cancer Management \& Research. 2011; 3: 57 .

7. Antoniou SA, Antoniou GA, Koch OO, Pointner R, Granderath FA. Laparoscopic colorectal surgery confers lower mortality in the elderly: a systematic review and meta-analysis of 66,483 patients. Surg Endosc. 2015; 29: 322-33.

8. Tegels JJ, Hulsewé KW, Sosef MN, Stoot JH. Morbidity and mortality after total gastrectomy for gastric malignancy: Do not forget about geriatric frailty and nutrition. Surgery. 2015; 157: 406-7.

9. Portanova M. Successful enteral nutrition in the treatment of esophagojejunal fistula after total gastrectomy in gastric cancer patients. World J Surg Oncol. 2010; 8: 71.

10. Nozoe T, Anai H, Sugimachi K. Usefulness of reconstruction with jejunal pouch in total gastrectomy for gastric cancer in early improvement of nutritional condition. Am J Surg. 2001; 181: 274-8

11. Biragyn A, Ferrucci L. Gut dysbiosis: a potential link between increased cancer risk in ageing and inflammaging. Lancet Oncol. 2018; 19: e295-e304.

12. Valdes AM, Walter J, Segal E, Spector TD. Role of the gut microbiota in nutrition and health. BMJ. 2018;361:k2179.

13. Lombardi VC, Meirleir KLD, Subramanian K, et al. Nutritional Modulation of the Intestinal Microbiota; Future Opportunities for the Prevention and Treatment of Neuroimmune and Neuroinflammatory Disease 4 . Macromol Biosci. 2018.

14. Zhao X, Chen $\mathrm{T}$, Meng $\mathrm{F}$, et al. Therapeutic effect of herb residue fermentation supernatant on spleen-deficient mice. Mol Med Rep. 2018; 17: 2764-70.

15. Chen T, Tian P, Huang $Z$, et al. Engineered commensal bacteria prevent systemic inflammation-induced memory impairment and amyloidogenesis via producing GLP-1. Appl Microbiol Biot. 2018: 1-11.

16. Chen H, Luo T, Chen T, Wang G. Seminal bacterial composition in patients with obstructive and non-obstructive azoospermia. Experimental \& Therapeutic Medicine. 2018; 15: 2884-90.

17. Tilg H, Adolph TE, Gerner RR, Moschen AR. The Intestinal Microbiota in Colorectal Cancer. Cancer cell. 2018.

18. Hersoug LG, Møller P, Loft S. Role of microbiota-derived lipopolysaccharide in adipose tissue inflammation, adipocyte size and pyroptosis during obesity. Nutr Res Rev. 2018: 1.

19. Gopalakrishnan V, Helmink BA, Spencer CN, Reuben A, Wargo JA. The Influence of the Gut Microbiome on Cancer, Immunity, and Cancer Immunotherapy. Cancer cell. 2018; 33: 570-80.

20. Crusell MKW, Hansen TH, Nielsen T, et al. Gestational diabetes is associated with change in the gut microbiota composition in third trimester of pregnancy and postpartum. Microbiome. 2018; 6: 89.

21. Cirstea M, Radisavljevic N, Finlay B. Good Bug, Bad Bug: Breaking through Microbial Stereotypes. Cell Host Microbe. 2018; 23: 10-3.

22. $\mathrm{Yu} X, \mathrm{Wu} \mathrm{X}, \mathrm{Qiu} \mathrm{L}$, et al. Analysis of the intestinal microbial community structure of healthy and long-living elderly residents in Gaotian Village of Liuyang City. Appl Microbiol Biot. 2015; 99: 9085-95.

23. Meng F, Chen T, Wang X, et al. Evaluation of the accuracy and sensitivity of high-throughput sequencing technology using known microbiota. Mol Med Rep. 2017.

24. Xin $\mathrm{F}$, Xin $\mathrm{W}$, Yang $\mathrm{S}$, et al. Evaluation of the Microbial Diversity in Amyotrophic Lateral Sclerosis Using High-Throughput Sequencing. Front Microbiol. 2016; 7.

25. Xu J, Lian FM, Zhao LH, et al. Structural modulation of gut microbiota during alleviation of type 2 diabetes with a Chinese herbal formula. Isme J. 2015; 9: 552-62.

26. Bolger A, Lohse M, Usadel B. Trimmomatic: a flexible trimmer for Illumina sequence data. Bioinformatics. 2014; 30: 2114-20.

27. Edgar R. UPARSE: highly accurate OTU sequences from microbial amplicon reads. Nat Methods. 2013; 10: 996-8.

28. Steege HT. Measuring Biological Diversity. Environ Ecol Stat. 2004; 1: 95-103.

29. Caporaso JG, Kuczynski J, Stombaugh J, et al. QIIME allows analysis of high-throughput community sequencing data. Nat Methods. 2010; 7: 335.

30. Osborn L. Leukocyte adhesion to endothelium in inflammation. Cell. 1990; 62: 3-6.

31. Paul WE, Seder RA. Lymphocyte responses and cytokines. Cell. 1994; 76: 241-51.

32. Zhou L, Sonnenberg GF. Essential immunologic orchestrators of intestinal homeostasis. Science Immunology. 2018; 3: eaao1605.

33. Postler TS, Ghosh S. Understanding the Holobiont: How Microbial Metabolites Affect Human Health and Shape the Immune System. Cell Metab. 2017; 26: 110.

34. Kramer CD, Genco CA. Microbiota, Immune Subversion, and Chronic Inflammation. Front Immunol. 2017; 8

35. Hand TW, Vujkoviccvijin I, Ridaura VK, Belkaid Y, Linking the microbiota, chronic disease and the immune system. Trends Endocrin Met. 2016; 27: 831.

36. Dwivedi M, Ansarullah, Radichev I, Kemp EH. Alteration of Immune-Mechanisms by Human Microbiota and Development and Prevention of Human Diseases. J Immunol Res. 2017; 2017: 1-2.

37. Forbes JD, Domselaar GV, Bernstein CN. The Gut Microbiota in Immune-Mediated Inflammatory Diseases. Front Microbiol. 2016; 7. 
38. World Health Organization. Dept. of Immunization V, Biologicals. Initiative for Vaccine Research: 2004-2005 Strategic Plan. Oral Surg Oral Med O. 2003; 95: 206-7.

39. Derrien M, Belzer C, de Vos WM. Akkermansia muciniphila and its role in regulating host functions. Microb Pathog. 2017; 106: 171-81. 